\section{SM Gerontology and Geriatric Research}

\section{Article Information}

Received date: Aug 03, 2018

Accepted date: Aug 28, 2018

Published date: Sep 03, 2018

\section{Corresponding author(s)}

Katherine Ka Pik Chang, Lecturer, School of Nursing, The Hong Kong Polytechnic University, Hunghom, Hong Kong, Tel: 852 27666324; Email: katherine.chang@polyu.edu.hk

Distributed under Creative Commons CC-BY 4.0

Keywords Telephone follow-up; Heart disease; Elderly

Article DOI 10.36876/smggr.1017

\title{
Content Analyses of Telephone Follow- up of Older People with Chronic Heart Problems using the Omaha System
}

\author{
Katherine Ka Pik Chang* and Frances Kam Yuet Wong \\ School of Nursing, The Hong Kong Polytechnic University, Hong Kong
}

Abstract

Background: This study is a secondary analysis of the process of telephone follow-up of communitydwelling older people with chronic heart disease after index hospitalization.

Objective: The objectives of the study were to understand the common problems encountered and the interventions provided by telephone, using the Omaha System as a framework.

Methods: The intervention nurse conducted assessments and interventions based on the system. Patient problems were categorized according to the system. Thirty-four sets of audio recordings, with a total of 896 minutes of verbatim from 17 participants, were transcribed and analyzed.

Results: The results identified the 5 most frequent problems and described the interventions most frequently prescribed by the intervention nurse. Circulation was the only physiological problem among the top problems identified. Nutrition, medication regime, physical activity and healthcare supervision were the common healthrelated behavioral problems cited at the initial and final call. Surveillance was the most frequent category of intervention, followed by teaching, guidance and counseling as the second most frequent category of intervention provided to the participants in the study.

Conclusions: A better understanding is needed of the process of care provided by the intervention nurse via telephone to older people with chronic heart disease in the community. The body of knowledge can extend the continuum of care provided to people living with chronic heart disease in the community. Further study on the integration of low and high technology would enhance the health surveillance and counseling of older people with chronic heart disease.

\section{Background}

Telephone support plays a significant role in providing continued care to patients after hospital discharge. Studies have reported an increase in the contribution of nurses to empowering people to self-manage their chronic health problems. A systematic review indicated that at least $50 \%$ of telephone calls can be handled by health advice alone, without the need for immediate healthcare attention in hospital [1].

There is a growing use of telephone support as an intervention for people with chronic heart problems. The low technology enhances accessibility, enabling people to receive continued care in a manner that is acceptable to them [1]. Decreasing mortality and or readmission rates, shortening length of stay, improving health-related quality of life, and reducing the intervention costs of people with chronic heart problems have commonly been examined in telephone support studies [2]. Most telephone support studies have focused on outcome effects. Future studies should focus on the process of proven interventions delivered by telephone support to people with chronic heart disease [2].

Heart disease is projected to be one of the three leading causes of burden of disease in 2030 [3]. It remained top among listed hospital discharge cases between 2000 and 2010 [4]. It is one of the most common chronic diseases and is associated with frequent hospital readmissions worldwide [5].

Chronic heart disease cannot be cured. It is not uncommon for people with heart disease to be readmitted to hospital. Being readmitted within one year after the first admission was one of the risk predictors of readmission for people aged 65 or over with heart disease [6]. Hospital readmissions of older people with chronic heart disease can be prevented if interventions are performed to address early signs and symptoms related to the circulatory system [7-9] and the behavioral factors can be adjusted [7].

Hospital-to-home transitions among older people require them to possess skills in self-managing their own medical condition. Patients are expected to comply with disease-specific interventions such as the adjustment of unhealthy lifestyle habits, medication and diet management, symptom 
management and coping with the disease [10]. Self-managing of one's medical condition is a proactive process [5] involving decision making and taking action with regard to one's medical condition. The process relies on the availability of accurate health information and useful advice from healthcare providers. The accessibility of healthcare providers to community-dwelling older people should be efficient.

\section{Omaha system}

There are increasing reports of the adaption of the Omaha System as a framework to support the delivery of care in the community. The system has been used for outcome measures on admission to and discharge from home care. Fifty-six studies were analyzed in a systematic review to evaluate the use of the Omaha System in practice [11]. Common outcome measures, including morbidity, mortality, satisfaction of patient and caregiver, and healthcare cost, were found to be effective. Little is known on the process of care.

Patient problems are categorized according to the system, which has 4 dimensions: environmental, psychosocial, physiological and health-related behaviors [12]. Each problem has a cluster of signs and symptoms that are unique to that problem. Interventions were identified according to 4 areas: Teaching, Guidance, and Counseling (TGC); Treatments and Procedures (TP); Case Management (CM); and Surveillance (S). TGC refers to activities designed to provide information and encourage self-managing action and coping. TP refers to practical activities such as relieving? People's signs and symptoms, prescriptions of medication, resistive exercises, and so forth. CM refers to activities that guide people toward the use of appropriate services. $S$ refers to activities that monitor and identify people's status in relation to a given condition.

\section{Main Study}

A transitional care program for community-dwelling old people with chronic health problems was evaluated [13]. The intervention nurse visited old people after the index admission. Readmission rates, quality of life, self-efficacy and global health condition were used to measure the effect of the transitional care program among older people discharged from hospital. The participants were randomized into three groups: an intervention group (home visit and telephone follow-up), a call group (telephone follow-up) and a control group (usual care). The transitional care model employed home visits and telephone calls to assist the delivery of interventions in the study, using the Omaha System as a framework for documentation. The intervention nurse conducted assessments and interventions based on the system. The interaction of older people with the intervention nurse facilitated the conduct of health assessments during home visits. The conveyance of health advice was maintained through follow-up with the older people via telephone. The collective methods had a significant effect in improving self-efficacy and quality of life and reducing hospital readmission. Significance differences in readmission were found between the intervention and control groups. Lower readmission rates were found in the intervention and call groups, but not in the control group. Older people in the intervention and call groups also benefited from the program. Among the participants who were living with different chronic diseases, most had chronic heart disease. A better understanding of the process of telephone support for the participants might contribute to advancements in the provision of transitional care.

\section{Objective}

This study used the data collected from our original study, which examined the effect of nurse follow-up after patient discharge from hospital for transitional care. The previous study had no plan to analyze the process of nurse-patient interaction during telephone follow-up. Our preliminary examination of the data suggests that there is value in exploring the interaction between nurses and older people with heart problems during telephone follow-up [13]. Using the Omaha System as a guide to assess, intervene and evaluate the intervention provided, the study nurse could follow up with the participants by telephone. The aim of the paper is to understand the problems identified and interventions implemented by nurses during telephone follow-up with community-dwelling older people with chronic heart problems.

\section{Methods}

This was a secondary analysis of the data collected in the main study. The data included 34 sets of audio recordings from 17 participants with heart disease during their telephone follow-up. The audio recordings were transcribed and written on a worksheet. The text contained in the transcript of the interaction between the intervention nurse and the participants was divided into units. A 'unit' was defined as the smallest phrase containing a single idea. A total of 1070 units were identified. Each unit was determined using the Omaha System. Twenty-one problem identification and intervention classifications were analyzed in each call of the study. Members of the coding team met and read through the transcripts in order to fully understand them. Members included a professor with expertise in transitional care, a lecturer whose previous study had included telephone follow-up, and a research assistant who had been trained in transcription based on the Omaha System. Intercoder reliability was monitored. Several meetings were held to ensure a common understanding of the units. The potential difficulties of transcription were also discussed during meetings. During content analysis, each unit was assigned to domains and subcategories of problems, categories and intervention targets. Random checks on the coding of selected samples were conducted to ensure inter-rater reliability. Direct agreement among members was maintained at between $97 \%$ and $100 \%$.

\section{Results}

The age range of the participants was from 62 to 91 years old. The time spent on the initial call ranged from 4 minutes to 51 minutes and 20 seconds. On the final call, the time spent ranged from 3 minutes and 23 seconds to 45 minutes and 40 seconds.

\section{Problems identified at initial and final calls}

There were 82 problems identified among the participants that required intervention at the initial and final calls in this study. At the initial call, circulation was the most frequent problem identified. It accounted for $32 \%$, followed by nutrition (25\%) and medication regimen (18\%). Physical activity and healthcare supervision were equal, each mentioned by $5 \%$ of participants. At the final call, circulation was the most frequent problem, accounting for $36 \%$ of the problems identified. Nutrition and medication regimen accounted for $12 \%$ and $11 \%$ of the problems respectively. Bowel function accounted for $9 \%$ and healthcare supervision for $8 \%$ of the problems 
Table 1: The top 5 problems identified using the Omaha System at initial and final calls

\begin{tabular}{|r|c|c|c|c|c|}
\hline \multirow{2}{*}{ Domain } & Problem & \multicolumn{3}{|c|}{ Rank (Frequency) } \\
\hline & & \multicolumn{3}{|c|}{ Initial Call } & \multicolumn{1}{|c|}{ Final Call } \\
\hline Physiological & Circulation & 1 & $(180)$ & 1 & $(136)$ \\
\hline Health-related behaviors & Nutrition & 2 & $(141)$ & 2 & $(46)$ \\
\hline Health-related behaviors & Medication regimen & 3 & $(100)$ & 3 & $(43)$ \\
\hline Health-related behaviors & Physical activity & 4 & $(30)$ & $\#$ & $(23)$ \\
\hline Health-related behaviors & Healthcare supervision & 5 & $(29)$ & 5 & $(29)$ \\
\hline Physiological & Bowel function & $\wedge$ & $(21)$ & 4 & $(36)$ \\
\hline & Sub-total & & $(501)$ & & $(313)$ \\
\hline & Others & & $(145)$ & & $(111)$ \\
\hline & Total & & $(646)$ & & $(424)$ \\
\hline
\end{tabular}

$\wedge$ Ranked $6^{\text {th }}$ in initial call

\# Ranked $7^{\text {th }}$ in final call

identified by the heart patients at the final call. Table 1 shows the 5 most common problems identified at the initial and final calls via telephone during follow-up.

Signs and symptoms of problems identified at initial and final calls

A total of 646 signs of problems were identified at the initial call. The common signs and symptoms of circulation problems were edema (cited 74 times), blood pressure (48 times) and irregular heart rate (25 times). The potential risks of not following the recommended nutrition plan (69 times); the lack of established standards for daily caloric/fluid intake (19 times); and overweight: adult BMI 25 or more (14 times) were the common signs and symptoms related to nutrition problems of participants in the study. The potential risk of not following the recommended dosage/schedule was the major problem of the medication regimen, sedentary physical activity (24 times) and healthcare supervision (22 times) of the participants identified at the initial call.

Table 2: The top 5 problems identified, with signs and symptoms at the initial and final calls.

\begin{tabular}{|c|c|c|c|}
\hline Problem & Signs and symptoms & \multicolumn{2}{|c|}{ Frequency } \\
\hline Circulation & Edema & 74 & 33 \\
\hline Initial call & Final call \\
\hline Nutrition & $\begin{array}{c}\text { Non-compliance with nutrition plan } \\
\text { (potential) }\end{array}$ & 69 & 40 \\
\hline $\begin{array}{c}\text { Irregular heart rate } \\
\text { regimen }\end{array}$ & $\begin{array}{c}\text { Lacks established standards for } \\
\text { daily caloric/fluid intake }\end{array}$ & 19 & 6 \\
\hline Onerweight (BMI > 25) & 14 & 1 \\
\hline $\begin{array}{c}\text { Onsical activity } \\
\text { Healthcare } \\
\text { supervision }\end{array}$ & regimen (potential) & 90 & 41 \\
\hline Bowel function & Sedentary lifestyle & 24 & 17 \\
\hline
\end{tabular}

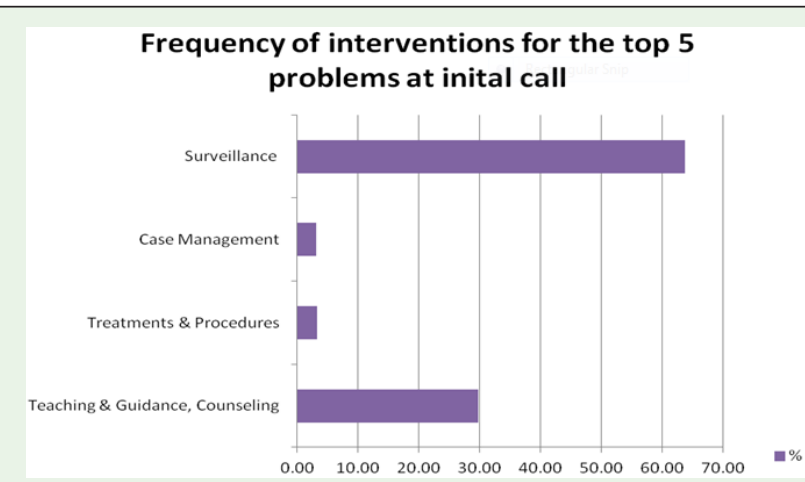

Figure 1: Frequency of interventions for the top 5 problems at initial call.

At the final call, 424 signs of problems were identified. High blood pressure (40 times) and edema (33 times) were the common signs and symptoms related to circulation problems. The potential risk of not following the recommend dosage/schedule (41 times) was a concern in terms of the medication regimen. The potential risk of not following the recommend nutrition plan (25 times) was a concern with regard to nutrition. The frequency of most of the signs and symptoms was reduced at the final call, except in the cases of healthcare supervision (25 times) and bowel functions (34 times). Constipation was the common problem of bowel function identified at final call. Table 2 presents the common signs and symptoms identified at the initial and final calls.

\section{Category of intervention identified at initial and final calls}

Problems identified at the initial and final calls were addressed by 4 categories of intervention. The number of interventions for the participants totaled 1072 at the initial and final calls. $60.4 \%$ of interventions were reported at the initial call, while $39.6 \%$ were reported at the final call. Surveillance was the major intervention implemented. Teaching, guidance and counseling was the second most frequent intervention type provided at the initial and final calls. Figures 1 and 2 illustrate the frequency of the interventions implemented for the top 5 problems at the initial and final calls respectively.

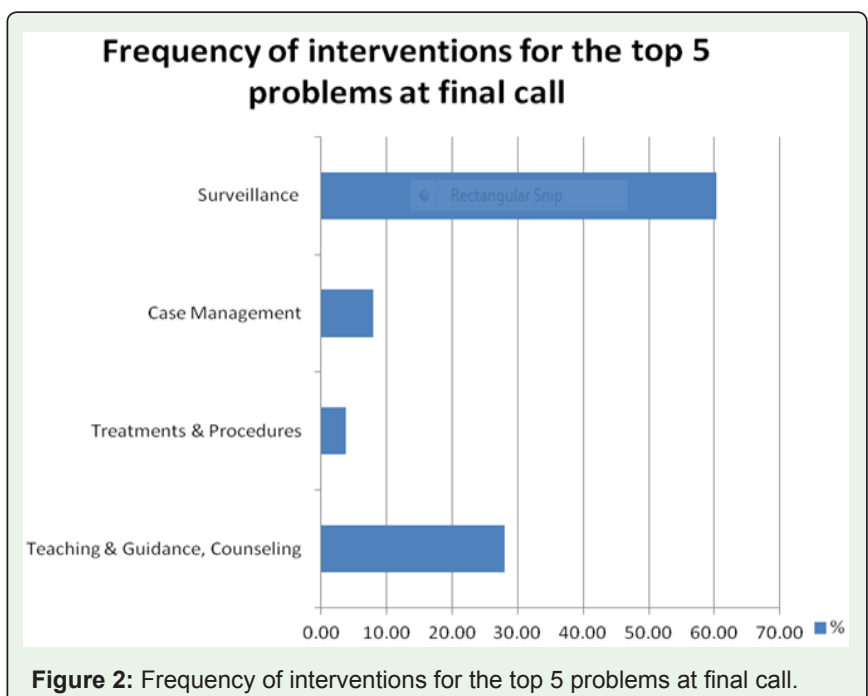


Table 3: The top 5 problems identified with interventions used at initial and final call

\begin{tabular}{|c|c|c|c|c|}
\hline \multirow[t]{2}{*}{ Domain } & \multirow[t]{2}{*}{ Problem } & \multirow[t]{2}{*}{ Intervention } & \multicolumn{2}{|c|}{ Frequency } \\
\hline & & & Initial call & Final call \\
\hline \multirow[t]{5}{*}{ Physiological } & Circulation & TGC & 61 & 37 \\
\hline & & TP & 8 & 6 \\
\hline & & $\mathrm{CM}$ & 2 & 4 \\
\hline & & S & 108 & 89 \\
\hline & & Total & 179 & 136 \\
\hline \multirow[t]{5}{*}{$\begin{array}{c}\text { Health-related } \\
\text { behaviors }\end{array}$} & Nutrition & TGC & 42 & 1 \\
\hline & & TP & 0 & 1 \\
\hline & & $\mathrm{CM}$ & 0 & 1 \\
\hline & & S & 99 & 26 \\
\hline & & Total & 141 & 46 \\
\hline \multirow[t]{5}{*}{$\begin{array}{c}\text { Health-related } \\
\text { behaviors }\end{array}$} & Medication regimen & TGC & 23 & 13 \\
\hline & & TP & 7 & 1 \\
\hline & & $\mathrm{CM}$ & 1 & 1 \\
\hline & & S & 69 & 28 \\
\hline & & Total & 100 & 43 \\
\hline \multirow[t]{5}{*}{$\begin{array}{c}\text { Health-related } \\
\text { behaviors }\end{array}$} & Physical activity & TGC & 11 & 7 \\
\hline & & $\mathrm{TP}$ & 0 & 1 \\
\hline & & $\mathrm{CM}$ & 0 & 0 \\
\hline & & S & 19 & 11 \\
\hline & & Total & 30 & 23 \\
\hline \multirow[t]{5}{*}{$\begin{array}{c}\text { Health-related } \\
\text { behaviors }\end{array}$} & $\begin{array}{l}\text { Healthcare } \\
\text { supervision }\end{array}$ & TGC & 6 & 2 \\
\hline & & $\mathrm{TP}$ & 1 & 0 \\
\hline & & $\mathrm{CM}$ & 12 & 17 \\
\hline & & $\mathrm{S}$ & 10 & 10 \\
\hline & & Total & 29 & 29 \\
\hline
\end{tabular}

Category of Intervention: Teaching, Guidance, and Counseling (TGC) Treatments and Procedures (TP); Case Management (CM); Surveillance (S)

\section{Linking of interventions with the top 5 problems}

Surveillance was most frequently linked to problems of circulation, nutrition, medication regimen and physical activity at initial and final call. Teaching, guidance and counseling participants was the second most frequently linked to all the top problems at both calls, except the problem of healthcare supervision. Table 3 illustrates the frequency of the interventions used to address the identified problems.

\section{Telephonic assessment algorithm}

The existing data identified the positive contribution of intervention nurses in empowering older people with chronic heart problems to take part in actively managing their heart condition in the long term. Among the identified problems, 5 were identified as the common primary needs of people living with chronic heart problems. The reporting of common signs and symptoms of the primary problems and their related interventions shed some light on the development of an efficient telephonic assessment algorithm.

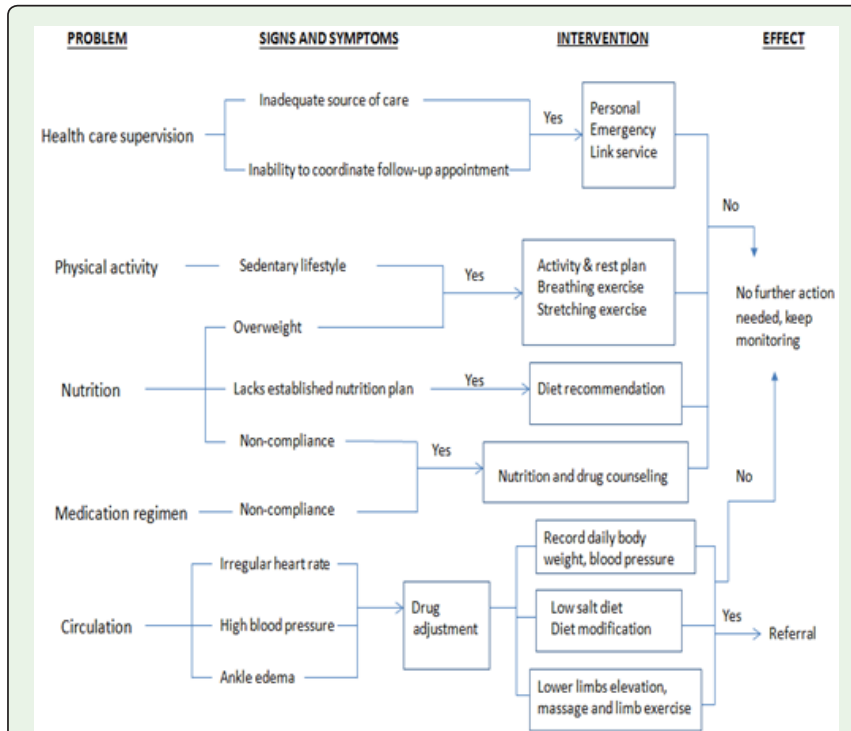

Figure 3: Focused telephone assessment for people with chronic heart problems using the Omaha System.

Figure 3 shows the focused telephonic assessment algorithm for people with chronic heart problems.

\section{Discussion}

The interaction between the intervention nurse and the older people in the study worked effectively. They shared problems and interventions during the calls. The establishment of a therapeutic relationship between the participants and the intervention nurse by telephone not only reduced dropout rates but also increased the participants' compliance with the interventions. Mutual agreement regarding the calling time to suit the participants' preferences was essential to ensure a good response rate for the telephone followup. Other strategies for establishing a therapeutic relationship by telephone included having the nurse give her/his own name and the name of the institution, greeting the participant by name, conscious use of tone, rate and volume of voice, and the appropriate use of language [14]. The intervention nurse checked with the participants consistently to ensure mutual understanding of the problems identified. This strategy enabled the nurse to offer the best possible care for the next call in the study.

\section{Care process using the Omaha System as a framework}

Circulation was the only physiological problem among the top problems identified at the initial call. It was prioritized as the top problem identified and addressed by the intervention nurse. The most frequently identified signs and symptoms of poor circulation were edema, high blood pressure and irregular heart rate. These signs and symptoms are commonly associated with increased risk of hospital readmission [15]. Nutrition, medication regime, physical activity and healthcare supervision were problems of health-related behaviors that were found to occur the most frequently at the initial and final calls. A different rank order of physical activity was found at the final call. The ranking of the health-related behaviors problem of physical activity was beyond the top 5 problems. Older people agreed that telephone calls encouraged them to stay physically active [16]. 
Results demonstrated an improvement in the identified problems after four telephone follow-ups. Although there were similar findings regarding the common problems identified at the initial call and those that had been addressed but still occurred at the time of the final call, the frequency of occurrence of the problems was found to have decreased. The frequency and duration of the telephone followup were optimal.

Care for a chronic medical problem requires numerous interventions. The success of the interventions requires a clear focus on implementation, commitment and the ability to make planned decisions to modify the intervention [17]. The decisionmaking process includes problem classification and intervention identification. Clinical judgment about whether people with chronic heart problems are progressing toward achieving their goals relies on data gathered. Gathering data is a continuous process. The Omaha System acts as a tool to guide the process of care by documenting the assessment, intervention and outcome evaluation. Problem identification includes 4 levels: the domains, problems, modifiers and signs and symptoms of the problem. In this study, the intervention nurse gathered relevant evidence before determining and prioritizing the problems requiring intervention. Records systematically facilitated the process of telephone follow-up [18]. She made use of the Omaha worksheet for documenting the care provided. Documentation of care makes visible the clinical judgment of the intervention nurse, highlighting the link between evidence of care and its outcomes.

\section{Surveillance as the first line of intervention}

Surveillance was the most frequent category of intervention, followed by teaching, guidance and counseling as the second most frequent category of intervention provided to the participants in this study. The findings were consistent with those of other studies $[19,20]$.

The intervention nurse monitored care and compared changes over time. Problems of circulation and nutrition were addressed by surveillance in most other studies [19]. In this study, potential noncompliance with the medication regime and physical activity were also addressed by surveillance. The risk factors for noncompliance with a medication regime included inadequate reading skills and poor visual acuity of medication labels. Such factors affecting the compliance of older people were consistently identified in another study [7]. In our study, the telephone acts as a tool to deliver verbal cues to older people regarding their diet regimen and medication compliance. Regular verbal cues could increase compliance behavior among older people [21] and encourage them to engage with their primary care providers [22]. The empowerment of older people in monitoring the signs and symptoms of their own circulation problems was found to be associated with reduced risk of hospital readmission [23].

\section{Improving health-related behavior via structured telephone support}

Teaching, guidance and counseling were commonly adopted by the intervention nurse in order to improve the health literacy of participants with chronic heart problems in our study. Low health literacy has been indicated as a risk factor for poor health-related outcomes and increased health service utilization, such as accident and emergency visits and hospital readmissions [24]. Another study suggests that repeated teaching and counseling provided by a nurse empowered lifestyle modifications and self-management of people with heart disease, reducing unnecessary utilization of hospital services [25]. In our study, the intervention nurse adopted an easily understandable approach to explain the relationship between heart dysfunction and the presence of circulation problems. Actions and side effects of medication, restriction of fluids with the presence of pitting edema, avoidance and recommended diet, and recommended physical activity were the common prescriptions in the domain of TGC. Confidence in the self-management of one's own health can be promoted.

\section{Standardization of telephone care process}

Comprehensive documentation of care provided using the telephone facilitates decision-making and the implementation of interventions based on identified problems. A telephonic assessment algorithm for people with chronic heart problems was formulated using the Omaha System in this analysis. Prediction of the level of risk could be linked to the related categories of intervention. Common lifestyle interventions that are associated with a reduced risk of heart problems, such as maintaining body mass index, limiting salt intake, and being active during one's leisure time [26], were included in the protocol.

\section{Possibility of using information technology}

People with chronic heart disease often experience a wide range of related symptoms that require immediate attention. Rapid response to those signs and symptoms of worsening heart problems during telephone follow-up is vital after index hospitalization. Subjective symptoms were detected and addressed by telephone in our study. Older people who are unable to report their symptoms precisely might not benefit fully from telephone follow-up. There is an increasing number of studies on adopting health technology to assist in the health surveillance of older people in the community. The application of technology in health plays a significant role in the continuum of care [27]. Further study should be conducted on the effect of adopting store-and-forward technologies with telephone follow-up.

\section{Conclusion}

In this study, the telephone was used as a medium for providing an immediate response in symptom management and in promoting compliance with health-related behavioral problems. The analysis not only enriches our knowledge of the common nature of problems encountered by older people with chronic heart disease after index hospitalization, but also increases our understanding of interventions in response to common problems identified during telephone followup. The body of knowledge can advance the continued care provided to people living with chronic heart disease in the community. Further study on the integration of low and high technology could enhance the health surveillance and counseling of older people with chronic heart disease.

\section{References}

1. Bunn F, Byrne G, Kendall S. The effects of telephone consultation and triage on healthcare use and patient satisfaction: A systematic review. British Journal of General Practice. 2005; 55: 956- 961. 
2. Inglis SC, Clark RA, McAlister FA, Ball J, Lewinter C, Cullington D, et al. Structured telephone support or telemonitoring programmes for patients with chronic heart failure (Review). Cochrane Database of Systematic Reviews. 2010.

3. Mathers $C D$, Loncar D. Projections of global mortality and burden of disease from 2002 to 2030. Journal of the Public Library of Science. 2006; 3: 2011 2030.

4. Go AS, Mozaffarian D, Roger VL. Heart disease and stroke statistics 2013 update: A report from the American Heart Association. Circulation. 2013; 127 e6-e245.

5. Riegel B, Dickson VV. A situation-specific theory of heart failure self-care. Journal of Cardiovascular Nursing. 2008; 23: 190-196.

6. Krumholz H, Chen YT, WangY, Vaccarino V, Radford MJ, Horwitz RI. Predictors of readmission among elderly survivors of admission with heart failure. American Heart Journal. 2000; 139: 72- 77.

7. McCauley KM, Bixby MB, Naylor MD. Advanced practice nurse strategies to improve outcomes and reduce cost in elders with heart failure. Disease Management. 2006; 9: 302-310.

8. Ehrenberg A, Ehnfors M, Ekman I. Older patients with chronic heart failure within Swedish community health care: A record review of nursing assessments and interventions. Journal of Clinical Nursing. 2003; 13: 90-96.

9. Suaya JA, Stason WB, Ades PA, Normand SL, Shepard DS. Cardiac rehabilitation and survival in older coronary patients. Journal of American College of Cardiology. 2009; 54: 25- 33

10. Tamparo CD, Lindh WQ. Therapeutic communications for health care. $3^{\text {rd }}$ ed Washington: Delmar Learning; 2008.

11. Topaz M, Golfenshtein N, Bowles KH. The Omaha system: A systematic review of the recent literature. J Am Med Inform Assoc. 2014; 21: 163-170.

12. Martin KS. The Omaha system: A key to practice, documentation, and information management. $2^{\text {nd }}$ ed. St. Louis: Elsevier Inc; 2005.

13. Wong FKY, Chow SKY, Chan TMF, Tam SKF. Comparison of effects between home visits with telephone calls and telephone calls only for transitional discharge support: A randomized controlled trial. Age and Ageing. 2014; 43 91- 97.

14. Josip C, Aziz S. Telephone consultations. British Medical Journal. 2003; 326: 966-969.

15. Dunlay SM, Gheorghiade M, Reid KJ, Allen LA, Chan PS, Hauptman PJ, et al. Critical elements of clinical follow-up after hospital discharge for heart failure: Insights from the EVERST trial. European Journal of Heart Failure. 2010; 12: 367- 374
16. Kolt GS, Oliver M, Schofield GM, Kerse N, Garrett N, Latham NK. An overview and process evaluation of TeleWalk: A telephone-based counseling intervention to encourage walking in older adults. Health Promotion International. 2006; 21: 201-208.

17. World Health Organization. Quality of care: A process for making strategic choices in health systems; 2006. Geneva WHO Press.

18. Males T. Telephone consultations in primary care: A practical guide; 2007 London: Royal College of General Practitioners.

19. Schoneman D. Surveillance as a nursing intervention: Use in community nursing centers. Journal of Community Health Nursing. 2002; 19: 33-47.

20. Brooten D, JoAnne M, Deatrick J, Naylor M. Patient problems, advanced practice nurse (APN) interventions, time and contacts among five patient groups. Journal of Nursing Scholarship. 2003; 35: 73- 79 .

21. Doggrell SA. Adherence to medicines in the older-aged with chronic conditions: Does intervention by allied health professional help? Drugs and Aging. 2010; 27: 239-254

22. Crocker JB, Crocker JT, Greenwald JL. Telephone follow-up as a primary care intervention for post discharge outcome improvement: A systematic review. 2012; 125: 915-921.

23. Coleman EA, Boult C. Improving the quality of transitional care for persons with complex care needs. Journal of the American Geriatrics Society. 2003; 51: $556-557$

24. Berkman ND, Sheridan SL, Donahue KE, Halpern DJ, Crotty K. Low health literacy and health outcomes: An updated systematic review. Annals of Internal Medicine. 2011; 155: 97-107.

25. Jerant AF, Azari $\mathrm{R}$, Martinez $\mathrm{C}$, Nesbitt $\mathrm{T}$. A randomized trial of telenursing to reduce hospitalization for heart failure: Patient-centered outcomes and nursing indicators. Home Health Care Services Quarterly. 2003; 22: 1- 20.

26. Del Gobbo LC, Kalantarian S, Imamura F, Lemaitre R, Siscovick DS, Psaty $B$, et al. Contribution of major lifestyle risk factors for incident heart failure in older adults: The Cardiovascular Health Study. J American CC: Heart Failure. 2015; 3: 520-528.

27. Coughlin JF, Pope JE, Leedle BR. Old age, new technology, and future innovations in disease management and home health care. Home Health Care Management \& Practice. 2006; 18: 196-207. 